\title{
HOMA-AD and Metabolic Syndrome-The Homecoming of Adiponectin in Pediatric Obesity
}

\author{
Anurag Bajpai ${ }^{1,2}$ \\ Received: 17 February 2021 / Accepted: 19 February 2021/ Published online: 11 March 2021 \\ (C) Dr. K C Chaudhuri Foundation 2021
}

A tremendous increase in the prevalence of childhood obesity over the last two decades is a major clinical challenge [1]. A substantial proportion of obese children and adolescents have metabolic complications posing a long-term health risk [2]. Their timely identification and management are highly desirable. Targeted intervention in at-risk individuals is preferable given the enormity of pediatric and adolescent obesity in the community.

Many obese children and adolescents do not develop metabolic complications despite high adiposity levels [3]. Identification of determinants of obesity complications may form the basis of a screening tool to identify individuals in the community with the greatest need for an intervention. The limited predictive value of anthropometric parameters for metabolic complications after a level of obesity suggests the need to explore alternate measures. Adiponectin, an adipocyte product and a correlate of insulin resistance, is a potential predictor for metabolic complications in obesity. Obese children have lower adiponectin levels than their normal-weight counterparts. Low adiponectin levels have been linked to metabolic complications; the association has, however, been variable [4]. The addition of insulin and glucose has been shown to enhance the predictive value of adiponectin. In this issue of the journal, Cândido et al. report the role of adiponectin, homeostatic model assessment-adiponectin (HOMA-AD), and HOMA-insulin resistance (HOMA-IR) in explaining metabolic complications in 691 Brazilian children and adolescents across body mass index categories [5]. In the study, HOMA$\mathrm{AD}$ (a measure of metabolic risk incorporating adiponectin,

Anurag Bajpai

dr_anuragbajpai@yahoo.com

1 Department of Pediatric \& Adolescent Endocrinology, Regency Center for Diabetes, Endocrinology \& Research, Regency City Clinic, Opposite PPN Market, Kanpur, Uttar Pradesh 208001, India

2 Center for Diabetes, Endocrinology and Cholesterol, Fortis Memorial Research Institute, Gurgaon, Haryana, India fasting blood glucose, and insulin levels) had the best diagnostic accuracy for predicting metabolic syndrome. A HOMA-AD level above 8.6 in children had a sensitivity of $80 \%$ and specificity of $92.7 \%$ to identify metabolic syndrome in children compared to $88.9 \%$ and $92.4 \%$ for levels above 14.3 for adolescents. The predictive value for adiponectin was substantially lower than that for HOMA-AD [4]. Regression analysis showed a greater dependence of HOMA-AD on metabolic risk factors than HOMA-IR or adiponectin levels.

The study reiterates the link between adiponectin and metabolic complications. The predictive value of HOMA-AD for metabolic complications suggests its role in metabolic screening; however the technical difficulties in its estimation limits its widespread use. The estimation of HOMA-AD requires fasting adiponectin, insulin, and glucose which makes its use in community settings difficult. Significantly, the predictive superiority of HOMA-AD over HOMA-IR for metabolic syndrome was restricted to adolescents with no advantage in children where HOMA-IR was better. This questions the role of adding another test on the cumbersome HOMA-IR estimation. Thus, the search for an easy-to-use, reliable, and valid tool to predict metabolic complications in the community continues.

\section{Declarations}

Conflict of Interest None.

\section{References}

1. NCD Risk Factor Collaboration (NCD-RisC). Worldwide trends in body-mass index, underweight, overweight, and obesity from 1975 to 2016: a pooled analysis of 2416 population-based measurement studies in 128.9 million children, adolescents, and adults. Lancet. 2017;390:2627-42.

2. Daniels SR. Complications of obesity in children and adolescents. Int J Obes (Lond). 2009;33(Suppl 1):S60-5. 
3. Dave C, Agarwal N, Patel R, Shukla R, Bajpai A. Predictors of metabolic complications in obese Indian children and adolescents. Indian J Pediatr. 2021;88(3):252-6.

4. Stroescu RF, Mărginean O, Bizerea T, Gafencu M, Voicu A, Doroș G. Adiponectin, leptin, and high sensitivity $\mathrm{C}$-reactive protein values in obese children - important markers for metabolic syndrome? J Pediatr Endocrinol Metab. 2019;32:27-31.
5. Cândido APC, Geloneze B, Calixto A, et al. Adiponectin, HOMAadiponectin, HOMA-IR in children and adolescents: Ouro Preto study. Indian J Pediatr. 2020. https://doi.org/10.1007/s12098-02003444-3.

Publisher's Note Springer Nature remains neutral with regard to jurisdictional claims in published maps and institutional affiliations. 